\title{
Outcomes of older patients aged 60 to 70 years undergoing reduced intensity transplant for acute myeloblastic leukemia: results of the NCRI acute myeloid leukemia
}

\section{6 trial}

Nigel H. Russell, ${ }^{1}$ Robert K. Hills, ${ }^{2}$ Abin Thomas, ${ }^{3}$ Ian Thomas, ${ }^{3}$ Lars Kjeldsen, ${ }^{4}$ Mike Dennis, ${ }^{5}$ Charles Craddock, ${ }^{6}$ Sylvie Freeman, ${ }^{7}$ Richard E. Clark ${ }^{8}$ and Alan K. Burnett ${ }^{9}$

${ }^{1}$ Department of Haematology, Nottingham University Hospitals, Nottingham, UK; ${ }^{2}$ Nuffield Department of Population Health, University of Oxford, Oxford, UK; ${ }^{3}$ Centre for Trials Research, College of Biomedical \& Life Sciences, Cardiff University, Cardiff, UK; ${ }^{4}$ Department of Haematology, Rigshospitalet, Copenhagen, Denmark; ${ }^{5}$ Department of Haematology, Christie Hospital, Manchester, UK; ${ }^{6}$ Department of Haematology, Queen Elizabeth Hospital,

Birmingham, UK; ${ }^{7}$ Department of Immunology and Immunotherapy, University of Birmingham, Birmingham, UK; ${ }^{8}$ Department of Molecular and Clinical Cancer Medicine, University of Liverpool, UK and ${ }^{9}$ Blackwaterfoot, Isle of Arran, UK

\author{
Correspondence: \\ Nigel Russell \\ nigel.russell@nottingham.ac.uk \\ Received: June 22, 2021. \\ Accepted: $\quad$ August 19,2021 \\ Prepublished: October 14, 2021. \\ https://doi.org/10.3324/haematol.2021.279010 \\ ○2022 Ferrata Storti Foundation \\ Haematologica material is published under a CC

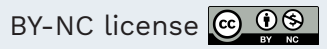

\begin{abstract}
Reduced intensity conditioning (RIC) transplantation is increasingly offered to older patients with acute myeloblastic leukemia. We have previously shown that a RIC allograft, particularly from a sibling donor, is beneficial in intermediate-risk patients aged 35-65 years. We here present analyses from the NCRI AML16 trial extending this experience to older patients aged 60-70 inclusive lacking favorable-risk cytogenetics. Nine hundred thirty-two patients were studied, with RIC transplant in first remission given to 144 (sibling $n=52$, matched unrelated donor $n=92$ ) with a median follow-up for survival from complete remission of 60 months. Comparisons of outcomes of patients transplanted versus those not were carried out using Mantel-Byar analysis. Among the 144 allografted patients, 93 had intermediate-risk cytogenetics, 18 had adverse risk and cytogenetic risk group was unknown for 33. In transplanted patients survival was $37 \%$ at 5 years, and while the survival for recipients of grafts from siblings (44\%) was better than that for recipients of grafts from matched unrelated donors (34\%), this difference was not statistically significant $(P=0.2)$. When comparing RIC versus chemotherapy, survival of patients treated with the former was significantly improved (37\% versus $20 \%$, hazard ratio $=0.67[0.53-0.84] ; P<0.001$ ). When stratified by Wheatley risk group into good, standard and poor risk there was consistent benefit for RIC across risk groups. When stratified by minimal residual disease status after course 1, there was consistent benefit for allografting. The benefit for RIC was seen in patients with a FLT3 ITD or NPM1 mutation with no evidence of a differential effect by genotype. We conclude that RIC transplantation is an attractive option for older patients with acute myeloblastic leukemia lacking favorable-risk cytogenetics and, in this study, we could not find a group that did not benefit.
\end{abstract}

\section{Introduction}

The outcome for older patients with acute myeloblastic leukemia (AML) has not shown the survival improvements that have been achieved in younger patients. ${ }^{1}$ The reasons for this include patient-related factors that can affect tolerance to the intensive chemotherapy regimens employed in younger patients but also the biological features of the disease. Patient-related factors include concurrent medical conditions, such as performance status and co-morbidities, which can adversely affect outcome.1,2 Disease-related characteristics include an increased incidence of poor-risk cytogenetics, ${ }^{3,4}$ an increased incidence of patients with secondary AML resulting from progression of an antecedent myelodysplastic syndrome ${ }^{1,4}$ and an increased expression of multidrug resistance mechanisms. ${ }^{5}$ As a consequence even when patients achieve a complete remission (CR) the risk of disease relapse remains high $^{1}$ and although over $65 \%$ of patients $>60$ years can achieve a CR with intensive chemotherapy approximately only $20 \%$ will survive to 5 years. ${ }^{6}$ Thus, interventions based on preventing relapse are a priority for development.

One therapeutic approach to reduce relapse is allogeneic stem cell transplantation. With the advent of RIC regimens, this therapy has been increasingly applied to older AML patients. Here the intention is to apply a cura- 
tive therapy but one that is known to have a significant risk of procedural mortality. In patients aged 40-59 years, a benefit for RIC over chemotherapy has been reported but in our experience and those of others, this was confined to sibling donors. ${ }^{7,8}$ A number of retrospective studies of registry data in patients over 60 years have been reported. The European Group for Blood and Marrow Transplantation reported a 4 -year survival of $27 \%$ in patients over 60 years with worse outcome in patients with poor-risk cytogenetics. ${ }^{9}$ A study from the Center for International Blood and Marrow Transplant Research reported similar results in older patients undergoing RIC in first CR (CR1). ${ }^{10}$ In that study multivariate analysis showed no impact of age on relapse, non-relapse mortality or survival. The Seattle consortium also confirmed that increasing age over 60 years was not associated with adverse outcomes whereas more co-morbidities and adverse cytogenetics were. ${ }^{11}$ A multicenter prospective phase II study also found a benefit with an overall survival at 2 years of $40 \% .^{12}$ Studies comparing RIC transplantation with standard chemotherapy in this age group are however lacking although recently the ALFA-1200 trial reported benefit in high-risk older patients only. ${ }^{13}$

The NCRI AML16 trial for patients with AML and high-grade myelodysplastic syndrome ( $>10 \%$ blasts) over the age of 60 years permitted RIC transplant in CR1 for patients with a suitably matched sibling or unrelated donor if they lacked favorable-risk cytogenetics. Here we report on a comparison of RIC transplantation with chemotherapy in older patients fit for intensive chemotherapy.

\section{Methods}

Between December 2006 and August 2012, 1,880 patients with AML or high-grade myelodysplastic syndrome (defined as $>10 \%$ blasts) from the UK and Denmark entered the NCRI AML16 trial (EUDRACT 2005-002847-14) for patients $>60$ years who were fit for intensive therapy. The trial schema and major outcomes have been published ${ }^{6}$ but, briefly, induction chemotherapy was daunorubicin/cytarabine or daunorubicin/clofarabine with or without a single dose of gemtuzumab ozogamicin $\left(3 \mathrm{mg} / \mathrm{m}^{2}\right)$ or daunorubicin/cytarabine with or without etoposide and with or without all-trans retinoic acid. Patients could be randomized between two or three courses of therapy and maintenance or not with azacytidine. Patients not in partial remission after course 1 were excluded from the randomization to two or three courses of therapy. The overall CR or incomplete CR (CRi) rate was $67 \%$ and did not differ between the arms. Patients in remission (CR or CRi) and who did not have favorable-risk disease could receive a RIC allograft from a matched sibling or matched unrelated donor (MUD) if considered fit for transplantation by the in- vestigator. Unrelated donor transplants were permissible if they were matched at HLA A, B, C, DRB1 and DQ at the allele level (10/10 HLA-match) or mismatched at a single locus (9/10 HLA-match). Details of the RIC conditioning regimens, which were not protocol-specified, are given in the Online Supplementary Appendix. Graft-versus-host disease prophylaxis and supportive care were in accordance with the local policy of the individual institutions. In this study a total of 148 transplants were performed in CR1 but as only four of these took place in patients $>70$ years this analysis is confined to the 144 patients aged 70 and less who did not have favorable-risk leukemia. Nine hundred thirty-two patients who had these characteristics were studied with a median follow-up from CR of 60 months. Of the 144 RIC transplants, 52 were from matched sibling donors and 92 from MUD. The characteristics of all the patients in this study are shown in Table 1.

The AML16 trial was approved by Council for Research Ethics Committees (COREC) and the local research ethics committee and conforms to local research governance procedures at each center. A copy of a center's local research ethics committee approval and site-specific assessment was lodged with the trial office at the University of Birmingham Clinical Trials Unit (BCTU) before entering patients.

\section{Statistical considerations}

Endpoint definitions follow the recommendations of the International Working Group..$^{14}$ Only transplantation in first remission is considered here; outcomes following transplantation or not are summarized using the Kaplan-Meier method and forest plots. The outcomes of the MUD and sibling transplant groups were compared using log-rank tests or Cox regression. When comparing the relapse risk, we considered death as a competing risk and a competing risk regression method was used for the analysis. For the comparison of transplant versus no transplant, to counteract the immortal time bias introduced by patients needing to have survived long enough to receive a transplant, Mantel-Byar methodology ${ }^{15}$ was used. Here all patients entering CR start in the "no transplant" group, with no patients in the transplant group. When a patient is transplanted, they change to the allograft group at the time of transplantation. This avoids early deaths counting against non-transplanted patients as transplanted patients must have survived long enough to receive a transplant and allows for the fact that MUD transplants tend to take place later than those from sibling donors. For the subgroup analysis using the forest plots, we tested for heterogeneity across all the subgroups with a test for trend wherever applicable. We summarize the characteristics of the patients across the group using frequencies and percentages for categorical data, and medians and quartile ranges for quantitative data. Comparisons of pa- 
tients' characteristics are based on $\chi^{2}$, Mantel-Haenszel tests for trend, or Wilcoxon rank sum tests as appropriate. All outcomes are summarized at 5 years of follow-up and statistical significance is set at $P<0.05$.

\section{Results}

\section{Patient characteristics}

Nine hundred thirty-two patients aged 60-70 inclusive who entered remission ( $\mathrm{CR}$ or $\mathrm{CRi}$ ) and did not have favorable-risk AML were studied with a median follow-up from remission of 60 months. A CONSORT diagram is shown in Online Supplementary Figure S1. Of the 144 RIC transplants performed, 52 were from matched sibling donors and 92 from MUD. The characteristics of all the patients in this study as well as those treated with chemotherapy and those treated with RIC are shown in Table 1. Patients selected for transplantation were more likely to be 65 years or younger and to have a better performance score. The transplant and non-transplant groups were balanced in terms of Wheatley risk groups which subdivides patients into three groups using a prospectively validated risk score comprising cytogenetics, white blood cell count, de novo or secondary AML, age and performance score. ${ }^{16}$ Of the transplant recipients, 83 were Wheatley good risk, 39 standard risk and 22 poor risk. Of the 144 RIC transplants, 52 were from matched sibling donors and 92 were from MUD. Ninety-three patients had intermediate-risk cytogenetics, 18 had adverse cytogenetics and cytogenetic risk status was unknown for 33. Of the 18 patients with adverse-risk cytogenetics, 15 (83.3\%) received transplants from an unrelated donor compared to $57 / 93$ (61.2\%) with intermediate-risk cytogenetics.

\section{Outcomes after reduced intensity conditioning transplants}

Detailed outcome data are provided in Table 2. The overall survival at 5 years after remission of the 144 recipients of a RIC transplant was $37 \%$ and was not significantly different between those with sibling (44\%) or unrelated (34\%) donors (unadjusted hazard ratio $[\mathrm{HR}]=1.35$ [0.85-2.13] $P=0.2$; adjusted for risk group: $H R=1.28$ [0.81-2.02] $P=0.3$ ) (Figure 1). Likewise, there was no significant difference in non-relapse mortality at 5 years between recipients of sibling (28\%) and MUD (36\%) transplants (unadjusted $\mathrm{HR}=1.45$ [0.78-2.73] $P=0.2$; adjusted for risk group: $H R=1.38$ [0.73-2.60] $P=0.3$; with relapse analyzed as a competing risk) (Online Supplementary Figure S2).

We also compared the outcome of sibling and MUD transplants by Wheatley risk group. This analysis showed no survival difference between sibling and MUD transplants for good ( $51 \%$ vs. $42 \%$ ), standard (49\% vs. $31 \%$ ) or poor risk (0\% vs. $13 \%)$ patients $(H R=1.26$ [0.8-2.00], test for trend
$P=0.7$ ) although relatively few sibling transplants (7 sibling, 15 MUD) were performed for poor-risk patients. The lack of evidence for a difference between outcomes of sibling and MUD RIC transplants led us to combine the two transplant types together for analyses comparing the outcome of transplantation or not.

Apart from survival, chronic graft-versus-host disease can have a major impact on post-transplant quality of life. The majority of patients in this study received in vivo T-lymphocyte depletion (Online supplementary File) and although quality of life outcomes were not collected, the incidence of severe chronic graft-versus-host disease at 12 months was only $12 \%$, was absent in $38 \%$, mild in $26 \%$ and moderate in $24 \%$.

\section{Comparison of reduced intensity conditioning transplantation with chemotherapyy}

When comparing RIC transplantation with no transplantation, survival was significantly improved by transplantation (37\% vs. 20\%, HR=0.67 [0.53-0.84], P<0.001) (Figure 2). Relapse-free survival was also improved by transplantation (32\% vs. $13 \%, \mathrm{HR}=0.56$ [0.45-0.70], $P<0.001$ ). The cumulative incidence of death in remission at 5 years among the transplant group was 34\% which compares to $10 \%$ with chemotherapy alone (unadjusted $\mathrm{HR}=5.02$ [3.46-7.29] $P<0.001$; adjusted for risk group: $\mathrm{HR}=5.02$ [3.46-7.29] $P<0.001$; with relapse analyzed as a competing risk) (Table 2 ).

To compensate for selection factors for transplant the comparison was repeated for the Wheatley risk groups. Consistent benefit for RIC allografting was seen in all risk groups with no evidence of any interaction ( $P$ value for trend 0.86) and the adjusted HR was 0.68 (0.54-0.85), $P<0.001$. (Figure 3 ) Overall survival at 5 years for patients in the Wheatley good-risk group was $45 \%$ vs. $26 \%$, standard risk $36 \%$ vs. $21 \%$ and poor risk $12 \%$ vs. $7 \%$ (Online Supplementary Figure $S 3 A-C$ ). Looking at age as a factor the survival of patients aged 60-65 (n=99) and 66-70 $(n=45)$ at 5 years undergoing RIC was $39 \%$ and $33 \%$ compared to $23 \%$ and $18 \%$ for those not transplanted ( $P$ value for heterogeneity 0.95) (Online Supplementary Figure S4). The benefit of RIC was seen in patients with intermediate risk, adverse risk or unknown risk cytogenetics (Online Supplementary Figure S5) and in those with a FLT3-ITD or NPM1 mutation with no evidence of a differential effect by genotype ( $P$ value for heterogeneity by subgroups 0.2 and 0.7 , respectively) although mutation results were not available for all patients (Figure 4). Relapse risk at 5 years was significantly reduced by transplantation (RIC 34\%, chemotherapy alone 77\%, $\mathrm{HR}=0.30$ [0.22-0.40] $P<0.001$; with death analyzed as a competing risk) (Table 2 and Online Supplementary Figure S6) and was not significantly different between recipients of sibling or MUD transplants when adjusted for risk group $(30 \%$ and $33 \%$ at 5 years, respectively, $P=0.9)$. 
Table 1. Demographics of 932 eligible patients in the AML16 reduced intensity conditioning study.

\begin{tabular}{|c|c|c|c|c|}
\hline Characteristics & Overall & No-allograft & Allograft & P-value \\
\hline N. of patients & 932 & 788 & 144 & \\
\hline $\begin{array}{l}\text { Age in years, } N(\%) \\
60-65 \\
66-70 \\
\text { Median (Q1-Q3) }\end{array}$ & $\begin{array}{l}480(51 \%) \\
452(49 \%) \\
65(63-68)\end{array}$ & $\begin{array}{l}381(48 \%) \\
407(52 \%) \\
66(63-68)\end{array}$ & $\begin{array}{c}99(69 \%) \\
45(31 \%) \\
64(62-66)\end{array}$ & $\begin{array}{l}<0.001 \\
<0.001^{\star *}\end{array}$ \\
\hline $\begin{array}{l}\text { Sex, } N(\%) \\
\text { Male } \\
\text { Female }\end{array}$ & $\begin{array}{l}543(58 \%) \\
389(42 \%)\end{array}$ & $\begin{array}{l}449(57 \%) \\
339(43 \%)\end{array}$ & $\begin{array}{l}94(65 \%) \\
50(35 \%)\end{array}$ & 0.062 \\
\hline $\begin{array}{l}\text { WHO PS(at diagnosis), N (\%) } \\
0 \\
1 \\
2 \\
3 \\
4\end{array}$ & $\begin{array}{c}578(62 \%) \\
306(33 \%) \\
28(3 \%) \\
18(2 \%) \\
2(<0.5 \%)\end{array}$ & $\begin{array}{c}469(60 \%) \\
273(35 \%) \\
27(3 \%) \\
17(2 \%) \\
2(<0.5 \%)\end{array}$ & $\begin{array}{c}109(76 \%) \\
33(23 \%) \\
1(1 \%) \\
1(1 \%) \\
0\end{array}$ & $<0.001^{\star}$ \\
\hline $\begin{array}{l}\text { Diagnosis, N (\%) } \\
\text { De novo } \\
\text { Secondary } \\
\text { High Risk MDS }\end{array}$ & $\begin{array}{l}691(74 \%) \\
134(14 \%) \\
107(12 \%)\end{array}$ & $\begin{array}{l}597(76 \%) \\
110(14 \%) \\
81(10 \%)\end{array}$ & $\begin{array}{l}94(65 \%) \\
24(17 \%) \\
26(18 \%)\end{array}$ & 0.012 \\
\hline $\begin{array}{l}\text { Cytogenetics, N (\%) } \\
\text { Intermediate } \\
\text { Adverse } \\
\text { Unknown }\end{array}$ & $\begin{array}{c}606(83 \%) \\
122(17 \%) \\
204\end{array}$ & $\begin{array}{c}513(83 \%) \\
104(17 \%) \\
171\end{array}$ & $\begin{array}{c}93(84 \%) \\
18(16 \%) \\
33\end{array}$ & 0.868 \\
\hline $\begin{array}{l}\text { White cell count } \times 10^{9} / \mathrm{L}, \mathrm{N}(\%) \\
0-9.9 \\
10-49.9 \\
50-99.9 \\
100+ \\
\text { Median }(\mathrm{Q} 1 \text { - Q3) }\end{array}$ & $\begin{array}{c}594(64 \%) \\
216(23 \%) \\
75(8 \%) \\
47(5 \%) \\
4.3(1.9-23.3)\end{array}$ & $\begin{array}{c}485(62 \%) \\
187(24 \%) \\
72(9 \%) \\
44(5 \%) \\
4.7(1.9-27)\end{array}$ & $\begin{array}{c}109(76 \%) \\
29(20 \%) \\
3(2 \%) \\
3(2 \%) \\
3.2(1.4-9.3)\end{array}$ & $\begin{array}{l}0.001^{*} \\
<0.001^{* *}\end{array}$ \\
\hline $\begin{array}{l}\text { Risk group, N (\%) } \\
\text { Good } \\
\text { Standard } \\
\text { Poor }\end{array}$ & $\begin{array}{l}547(49 \%) \\
271(29 \%) \\
204(22 \%)\end{array}$ & $\begin{array}{l}374(48 \%) \\
232(29 \%) \\
182(23 \%)\end{array}$ & $\begin{array}{l}83(58 \%) \\
39(27 \%) \\
22(15 \%)\end{array}$ & $0.013^{\star}$ \\
\hline $\begin{array}{l}\text { HCTCl score, } \mathrm{N}(\%) \\
0 \\
1 \text { or } 2 \\
3 \text { or more } \\
\text { Unknown }\end{array}$ & $\begin{array}{c}474(51 \%) \\
313(34 \%) \\
139(15 \%) \\
6\end{array}$ & $\begin{array}{c}390(50 \%) \\
267(34 \%) \\
126(16 \%) \\
5\end{array}$ & $\begin{array}{c}84(50 \%) \\
46(34 \%) \\
13(9 \%) \\
1\end{array}$ & $0.016^{\star}$ \\
\hline $\begin{array}{l}\text { ITD, N (\%) } \\
\text { WT } \\
\text { Mutant } \\
\text { Fail } \\
\text { Unknown }\end{array}$ & $\begin{array}{c}341(82 \%) \\
76(18 \%) \\
9 \\
506\end{array}$ & $\begin{array}{c}290(81 \%) \\
70(19 \%) \\
6 \\
422\end{array}$ & $\begin{array}{c}51(90 \%) \\
6(10 \%) \\
3 \\
84\end{array}$ & 0.105 \\
\hline $\begin{array}{l}\text { NPM1, N (\%) } \\
\text { WT } \\
\text { Mutant } \\
\text { Fail } \\
\text { Unknown }\end{array}$ & $\begin{array}{c}281(73 \%) \\
102(27 \%) \\
14 \\
535\end{array}$ & $\begin{array}{c}237(72 \%) \\
93(28 \%) \\
11 \\
447\end{array}$ & $\begin{array}{c}44(83 \%) \\
9(17 \%) \\
3 \\
88\end{array}$ & 0.087 \\
\hline $\begin{array}{l}\text { ITD/NPM1, N (\%) } \\
\text { WT/WT } \\
\text { WT/Mut } \\
\text { Mut/WT } \\
\text { Mut/Mut } \\
\text { Unknown }\end{array}$ & $\begin{array}{c}246(65 \%) \\
63(17 \%) \\
30(8 \%) \\
37(10 \%) \\
556\end{array}$ & $\begin{array}{c}207(64 \%) \\
57(18 \%) \\
26(8 \%) \\
35(11 \%) \\
463\end{array}$ & $\begin{array}{l}39(76 \%) \\
6(12 \%) \\
4(8 \%) \\
2(4 \%) \\
93\end{array}$ & 0.251 \\
\hline $\begin{array}{l}\text { Donor type, N (\%) } \\
\text { Sibling } \\
\text { MUD }\end{array}$ & & & $\begin{array}{l}52(36 \%) \\
92(64 \%)\end{array}$ & \\
\hline
\end{tabular}

All tests are the $\chi^{2}$-test, except: *Mantel-Haenszel test for trend and **Wilcoxon rank-sum test. Q1: first quartile; Q3: third quartile; WHO PS, World Health Organization performance status; MDS: myelodysplastic syndrome; WBC: white blood cell count; HCTCI: Hematopoietic Cell Transplantation Comorbidity Index; ITD: internal tandem duplication; WT: wild-type; Mut: mutated; MUD: matched unrelated donor 
On entry to AML16, all patients had their Hematopoietic 313 (33.6\%) and 3+ in 139 (14.9\%). For patients proceeding Cell Transplant Specific Co-Morbidity Index (HCT-Cl) to RIC transplantation the $\mathrm{HCT}$-Cl was available for measured. ${ }^{17}$ This measurement was available for 926/932 143/144 patients and the relative frequencies of scores 0 , patients who entered CR1 and was 0 in 474 (51.9\%), 1-2 in 1-2 and 3+ were 59\%, 32\% and 9\%, respectively. When sur-

Table 2. Survival estimates.

\begin{tabular}{|c|c|c|c|c|}
\hline Category & Transplant & No transplant & HR $(95 \% \mathrm{CI})$ & P-value \\
\hline & $N=144$ & $\mathbf{N}=932$ & & \\
\hline Overall survival after CR1 & $37 \%$ & $20 \%$ & $0.67(0.53-0.84)$ & $<0.001$ \\
\hline Relapse free survival & $32 \%$ & $13 \%$ & $0.56(0.45-0.70)$ & $<0.001$ \\
\hline $\mathrm{Cl}$ of death in remission $1^{* *}$ & $34 \%$ & $10 \%$ & $5.07(3.46-7.29)$ & $<0.001$ \\
\hline $\mathrm{Cl}$ of relapse* & $34 \%$ & $77 \%$ & $0.30(0.22-0.40)$ & $<0.001$ \\
\hline \multicolumn{5}{|c|}{ Survival by Wheatley risk group } \\
\hline Good risk & $45 \%$ & $26 \%$ & $0.65(0.46-0.90)$ & 0.009 \\
\hline Standard risk & $36 \%$ & $21 \%$ & $0.75(0.49-1.15)$ & 0.190 \\
\hline Poor risk & $12 \%$ & $7 \%$ & $0.66(0.41-1.06)$ & 0.058 \\
\hline \multirow[t]{2}{*}{ Category } & Sibling & MUD & HR (95\%CI) & P-value \\
\hline & $N=52$ & $\mathrm{~N}=92$ & & \\
\hline Overall survival after CR1 & $44 \%$ & $34 \%$ & $1.35(0.85-2.13)$ & 0.200 \\
\hline Relapse-free survival & $42 \%$ & $31 \%$ & $1.25(0.80-1.95)$ & 0.318 \\
\hline $\mathrm{Cl}$ of death in remission $1^{\star *}$ & $28 \%$ & $36 \%$ & $1.45(0.78-2.73)$ & 0.242 \\
\hline $\mathrm{Cl}$ of relapse* & $30 \%$ & $33 \%$ & $1.17(0.63-2.17)$ & 0.614 \\
\hline
\end{tabular}

*Death as a competing risk; ${ }^{* *}$ Relapse as a competing risk. HR: hazard ratio; $95 \% \mathrm{CI}: 95 \%$ confidence interval; CR1: first complete remission; $\mathrm{Cl}$ : cumulative incidence.

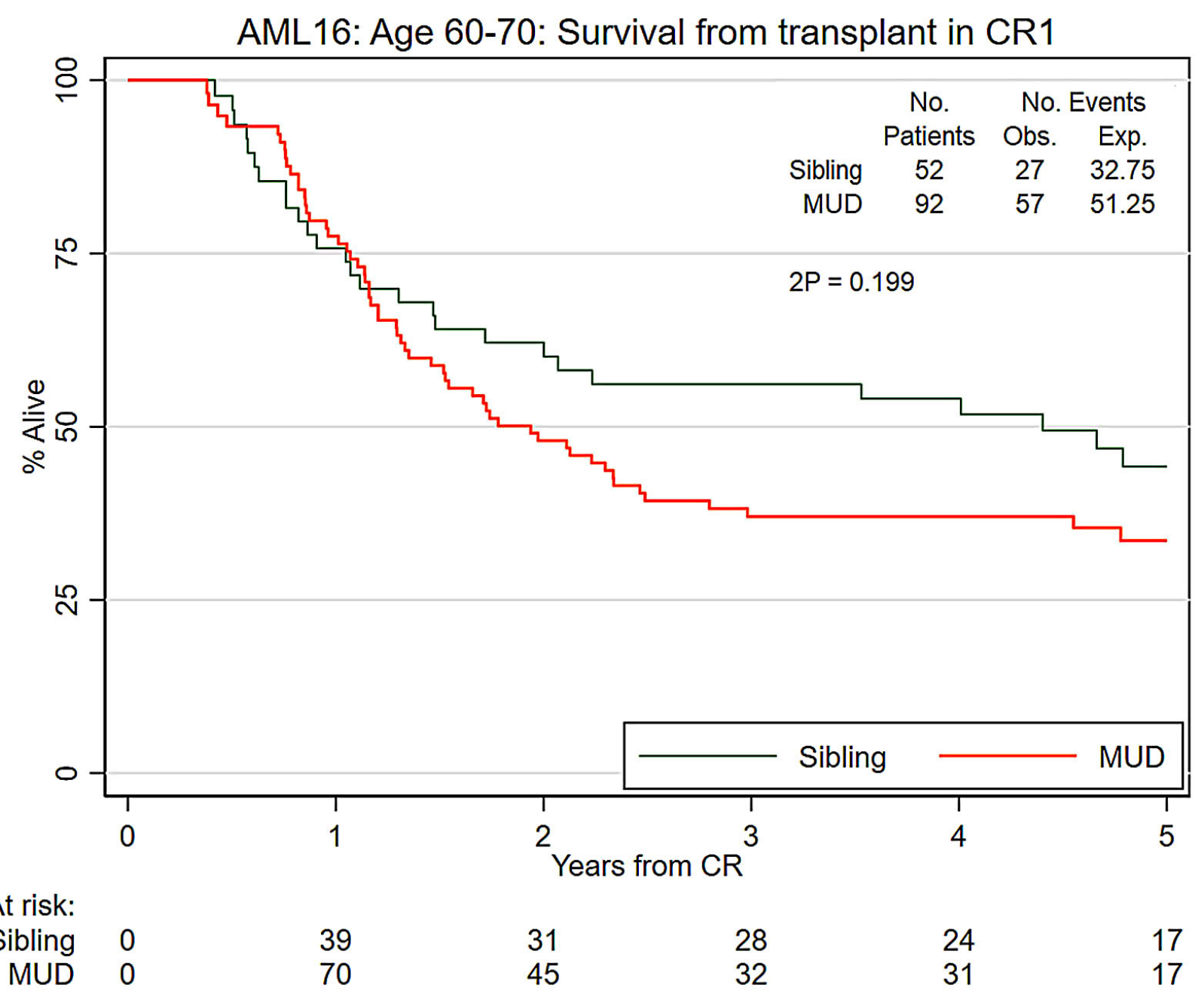

Figure 1. Survival after first complete remission comparing sibling and unrelated donors. CR1: first complete remission; Obs: observed; Exp: expected; MUD: matched unrelated donor; Allo_Type: type of allogeneic transplant. 
AML16: Age 60-70: Overall survival comparing RIC transplant or not in CR1

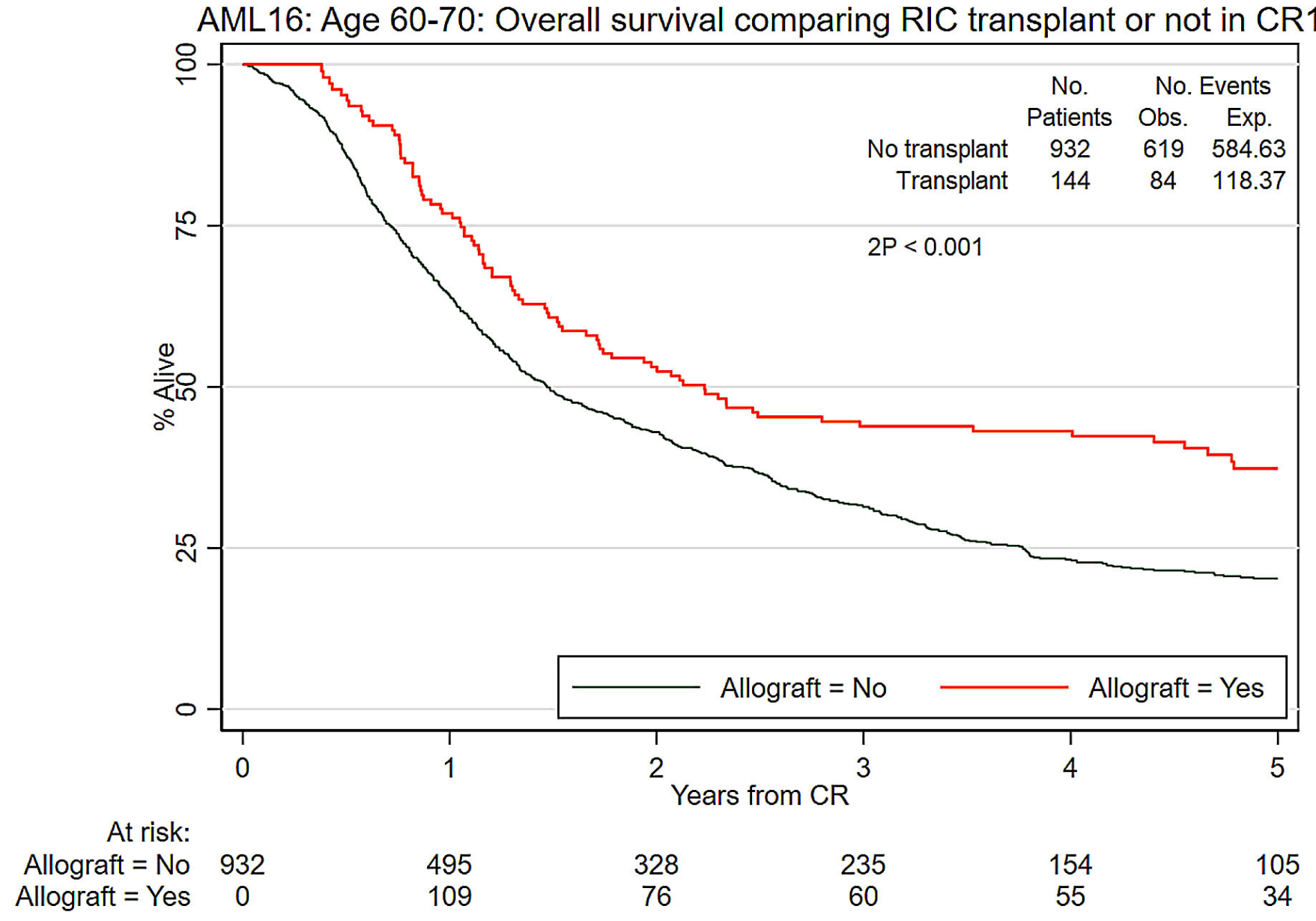

Figure 2. Overall survival comparing reduced intensity conditioning transplantation or not for acute myeloid leukemia in first complete remission. RIC: reduced intensity conditioning; CR1: first complete remission; Obs: observed; Exp: expected.

vival was analyzed by $\mathrm{HCT}-\mathrm{Cl}$ risk group we observed that all HCT-Cl risk groups had benefit ( $P$ value for heterogeneity by subgroups 0.08). Paradoxically there was a trend for increased benefit with higher HCT-CI ( $P$ value for trend 0.03) although numbers transplanted with an $\mathrm{HCT}-\mathrm{Cl}>3$ were small (Online Supplementary Figure S7).

\section{Impact of minimal residual disease after course 1 on transplant outcome}

Flow minimal residual disease (MRD) status after course 1 was available for 323 patients, as previously reported. ${ }^{18}$ These included 36 patients who went on to have a RIC transplant. When stratified by post-course 1 flow MRD status (MRD-negative, MRD unknown, MRD-positive and no CR/CRi after course 1) there was again consistent benefit for allografting. $(\mathrm{HR}=0.68$ [0.54-0.85] $P<0.001)$ with no heterogeneity $(P=0.20)$ (Figure 5$)$.

\section{Discussion}

Here we report on a large prospective evaluation of RIC transplantation compared to chemotherapy in AML patients aged 60-70 years inclusive in first remission who were considered fit for intensive treatment. Our experience shows that when comparing an allograft with no transplant, survival was significantly improved for the former with overall survival at 5 years of $37 \%$ compared to $20 \%$ for patients treated with chemotherapy. This bene- fit was present in patients with a good, intermediate or poor prognosis as stratified by the Wheatley index, which includes both disease- and patient-specific factors that can affect outcome. Therefore, this benefit was seen in those with adverse-risk features including secondary AML, adverse-risk cytogenetics and older age and was seen in those with a FLT3 ITD. The benefit from RIC transplantation was due to a significant reduction in the risk of relapse, which outweighed the negative impact of excess transplant-related mortality. These results are consistent with the results from our AML15 trial in which we showed a benefit for RIC transplantation for the age range 35-65 years although in that study only when sibling donors were used. ${ }^{7}$ In AML16 the benefit from transplantation was seen with both sibling and unrelated donors and although sibling transplants generally performed better, survival was not significantly different particularly as more patients with adverse-risk features received transplants from unrelated donors. Although in this study we adjusted for known factors only $15 \%$ of patients who entered remission underwent RIC so there might be other selection factors operative that could have biased the results. In our NCRI AML16 trial, we previously reported that MRD status after course 1 was the most important independent prognostic factor for relapse and survival, being independent of age, cytogenetics, white blood cell count, secondary disease and performance status. ${ }^{18}$ In this analysis we could not find any evidence of an interaction with treatment outcome following RIC and all groups appeared to 
AML16: Allograft in 1st remission Mantel-Byar analysis of survival by risk group

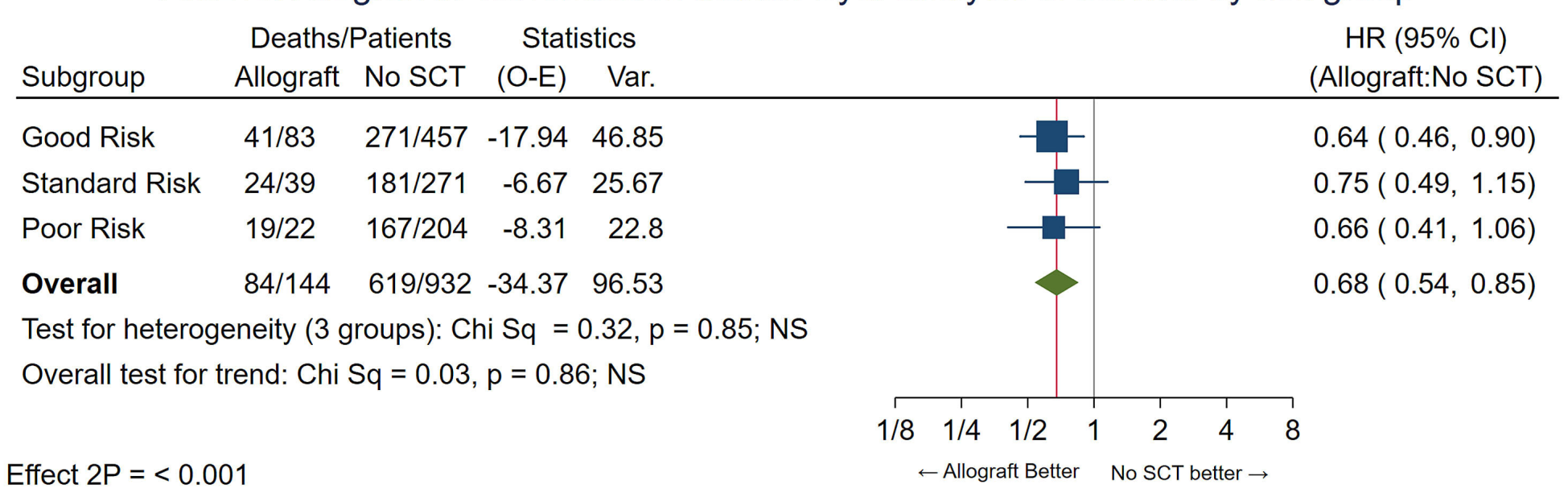

Figure 3. Mantel-Byar analysis of overall survival by Wheatley risk group. SCT: stem cell transplant; O-E: observed - expected; HR: hazard ratio; $95 \% \mathrm{Cl}$ : 95\% confidence interval.

AML16: Allograft in 1st remission Mantel-Byar analysis of survival by molecular status

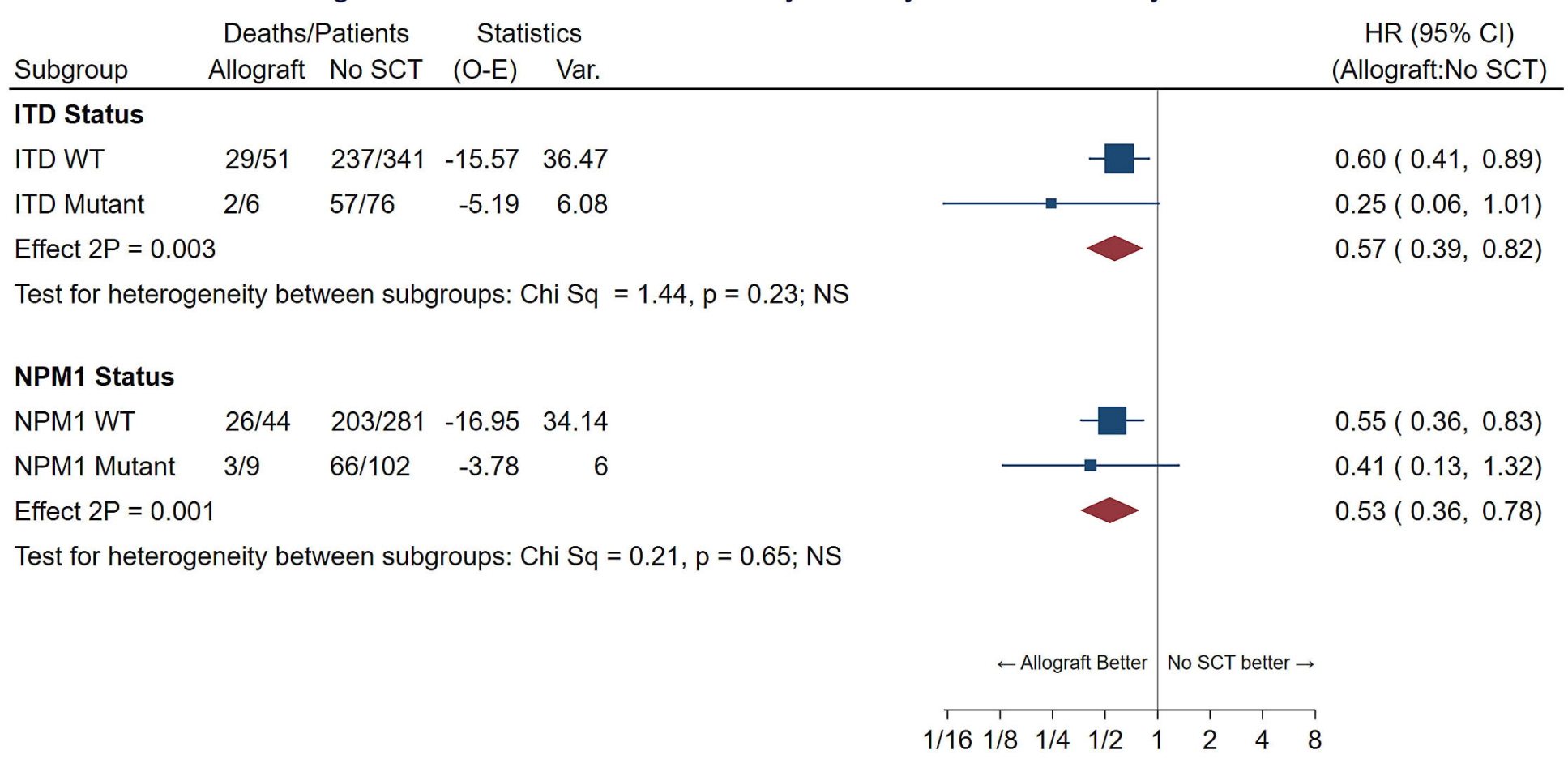

Figure 4. Mantel-Byar analysis of overall survival by FLT3/NPM1 status. SCT: stem cell transplant; O-E: observed - expected; HR: hazard ratio; 95\% Cl: 95\% confidence interval; ITD: internal tandem duplication; WT: wild-type.

AML16: Allograft in 1st remission Mantel-Byar analysis of survival by MRD status

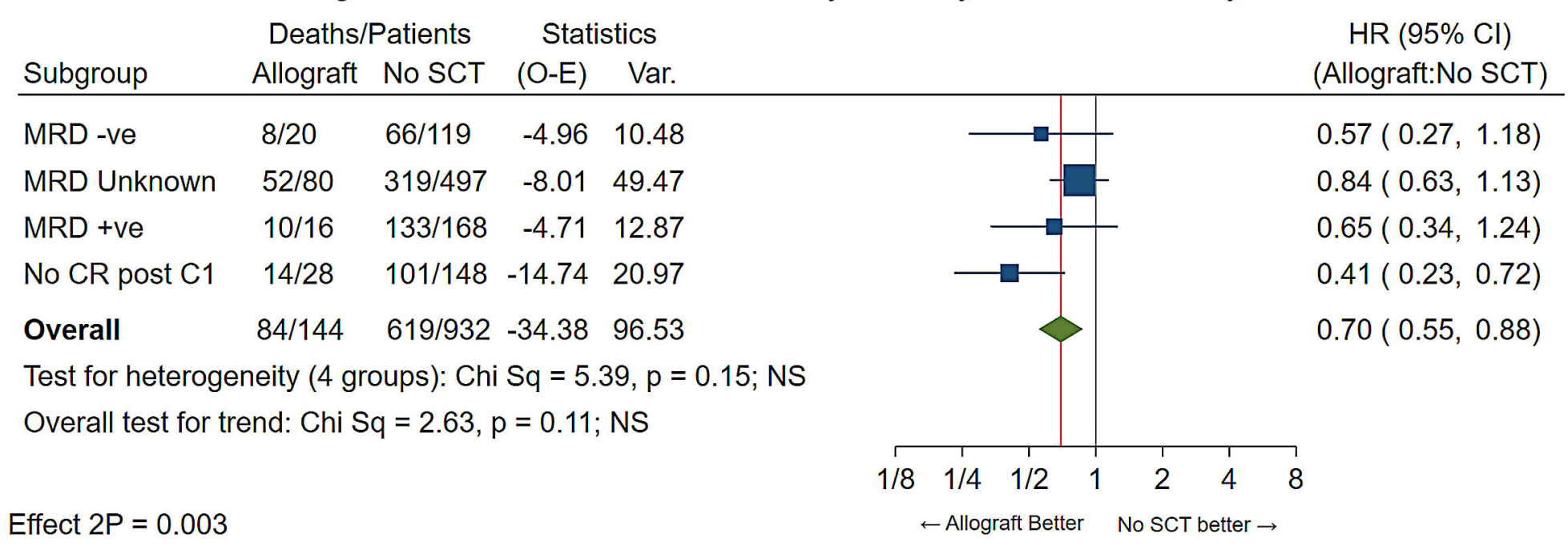

Figure 5. Mantel-Byar analysis of overall survival by minimal residual disease status after course 1. MRD: minimal residual disease; SCT: stem cell transplant; O-E: observed - expected; HR: hazard ratio; 95\% Cl: 95\% confidence interval; CR: complete remission; C1: course 1. 
benefit independently of their post-course 1 MRD status including those patients who were MRD-positive or had failed to achieve a CR with their first course of chemotherapy. MRD status was not re-assessed immediately pre-transplant and could have changed as a consequence of subsequent chemotherapy, so whether the benefit of transplantation in post-course $1 \mathrm{MRD}$-positive patients is confined to those becoming MRD-negative after course 2, is being studied in the NCRI AML18 trial. .

One factor that can affect outcome following an allogeneic transplant is the presence of co-morbidities with an HCT$\mathrm{Cl}$ score of $3+$ being associated with a significantly higher risk of non-relapse mortality. ${ }^{19}$ The majority of patients entering AML16 had an HCT-Cl of 0 or 1-2 and we could not detect an adverse impact of higher $\mathrm{HCT}-\mathrm{Cl}$ on outcome although the numbers of patients with a high score were small. Furthermore, our assessment of $\mathrm{HCT}-\mathrm{Cl}$ was made at trial entry not immediately pre-transplant so there could have been changes in the score of some patients as a consequence of receiving induction treatment. However, our findings do suggest that the majority of patients in this age group being treated with intensive chemotherapy are likely to have a health status reflected by a co-morbidity score of 3 or less which would not preclude them from being considered for RIC transplantation. Despite these findings, only a minority of patients underwent transplantation suggesting that this modality of therapy has been underused although transplant rates have increased to $31 \%$ in our current NCRI AML18 trial. ${ }^{20}$

In summary, our results show that while, as patients get older, there will inevitably be selection for treatments based on fitness, our analysis adjusted for the clinical features of the disease shows that RIC transplantation results in improved survival for older patients with AML. Despite these findings and considering the significant increase in transplant-related mortality associated with transplantation more precise risk stratification is required in the selection of older patients for transplants. This may be provided by more detailed genomic analysis particularly in intermediate-risk patients for whom it has been reported that the benefit of transplantation is limited to those with gene mutations typical of secondary AML. ${ }^{13}$

\section{Disclosures}

No conflicts of interest to disclose.

\section{Contributions}

$A K B$. NHR, and RKH conceived and designed the study; $A K B$, $N H R, C C, L K, R E C$, and SF provided study materials or patients; $A K B, R K H, N H R, C C, M D, L H, R E C, S F, I T$, and AT collected and assembled data; $A K B, R K H, N H R$, and $A T$ analyzed and interpreted the data; NHR drafted the paper which was revised and approved by all authors.

\section{Acknowledgments}

The authors would like to thank the Cardiff University Haematology Trials Unit staff for supervision of the trial and the following investigators: Aalborg University Hospital: Kallenbach; Aarhus University Hospital: Starklint, Norgaard, Friis, Steffensen, Moelle, Skov Holm; Aberdeen Royal Infirmary: Culligan, Tighe; Addenbrooke's Hospital: Craig, Crawley, Marcus; Arrowe Park Hospital: Butt, Dasgupta; Barnet General Hospital: Virchis; Basingstoke and North Hampshire Hospital: Simpson, Milne; Belfast City Hospital: Cuthbert, McMullin, Jones; Birmingham Heartlands Hospital: Milligan, Lovell, Paneesha, Smith, Pratt; Blackpool Victoria Hospital: Cahalin; Borders General Hospital: Okhandiar, Tucker; Bradford Royal Infirmary: Ackroyd, Williams, Newton; Bristol Haematology \& Oncology Centre: Standen, Evely, Mehta, Robinson, Bird, Robinson, Marks; Cheltenham General Hospital: Blundell, Rye, Lush, Robinson; Chesterfield \& North Derbyshire Royal Hospital: Wodzinski, Cutting; Christie Hospital: Dennis, Liakopoulou; Churchill Hospital: Vyas, Peniket; City Hospitals Sunderland: Pemberton, Marshall, Hervey, Lyons; Colchester General Hospital: Maboreke, Hamblin; Countess of Chester Hospital: Lee, Tueger; Crosshouse Hospital: Mccoll, Maclean; Croydon University Hospital: Osuji, Pollard; Derbyshire Royal Infirmary: McKernan, Smith; Derriford Hospital: Rule, Hunter, Nokes, Copplestone, Hamon; Doncaster Royal Infirmary: Kaul, Paul; Ealing Hospital: Abrahamson; Eastbourne District General Hospital: Grace; Falkirk Community Hospital: Neilson; Gartnavel General Hospital: Fitzsimmonds, Soutar, Drummond, McKay; Gloucestershire Royal Hospital: Chown, Frewin, MacPherson, Rye; Guy's Hospital: Carr, Smith; Hammersmith Hospital: Apperley; Hemel Hempstead General Hospital: Harrison, Wood; Hereford County Hospital: Robinson; Herlev University Hospital: Hoegh Dufva, Helleberg, Jensen; Hillingdon Hospital: Jan-Mohamed, Kaczmarski; Hull Royal Infirmary: Ali, Carter; Ipswich Hospital: Dodd, Ademokun; James Paget Hospital: Gomez; Kent \& Canterbury Hospital: Pocock, Saieed, Ratnayake, Zwaan, Lindsay; Kettering General Hospital: Kwan, Lyttleton; King's College Hospital (Denmark Hill): Lucas; Kingston Hospital: Sykes; Leicester Royal Infirmary: Hunter, Garg; Lincoln County Hospital: Saravanamuttu; Maidstone District General Hospital: Gale, Rassam, Gillett; Manchester Royal Infirmary: Lucas, Yin, Burthem, Tholouli; Medway Maritime Hospital: Aldouri, Andrews, Eden; Monklands Hospital: Mitchell, Patterson; Musgrove Park Hospital: Bolam, Mannari; New Cross Hospital: Basu, Jacob, Handa; Ninewells Hospital: Gelly, Tauro, Meiklejohn, Gowans, Marron; Norfolk \& Norwich University Hospital: Lawes; North Staffordshire Hospital: Chasty, Stewart; Northampton General Hospital (Acute): Mittal; Northwick Park Hospital: Panoskaltsis; Nottingham University Hospitals NHS Trust - City Hospital Campus: Das-Gupta, Russell, Byrne; Odense University Hospital: Friis; Pilgrim Hospital: Tringham, Saravanamuttu; Pinderfields General Hospital: 
Ashcroft, Moreton, Wright, Patil, Chapple; Poole General Hospital: Bell, Jack; Princess Royal University Hospital: Vadher, De Lord, Lakhani; Queen Alexandra Hospital: Dignum, Corser, Cranfield, Ganczakowski; Queen Elizabeth Hospital (Kings Lynn): Keidan, Coates; Queen Elizabeth Hospital Birmingham: Murray, Craddock, Mahendra, Cook; Queen Elizabeth Hospital, Woolwich: Cheung; Queen's Hospital, Romford: Grant, Brownell, Stevens, Hemmaway; Raigmore Hospital: Forsyth, Lush; Rigshospitalet University Hospital: Kjeldsen, Jensen, Nielsen, Dimitrijevic, Bjerrum, Niemann, Schoelkopf, Friis, Kampmann, Groenbaek; Rotherham District General Hospital: Barker, Taylor; Royal Berkshire Hospital: Mucklow, Ramasamy, Simpson; Royal Bournemouth General Hospital: Killick, Hall, Walewska; Royal Cornwall Hospital (Treliske): Pottinger, Parkins, Creagh, Royal Devon \& Exeter Hospital (Wonford): Hamilton, Joiner, Lee, Ruell, Todd, Rudin, Coppell, Kerr, Royal Free Hospital: Kottaridis, Mehta, McNamara, Royal Gwent Hospital: Jenkins, Jackson, Osman, Royal Hallamshire Hospital: Dalley, Reilly, Snowden; Royal Stoke University Hospital (University Hospital of North Midlands NHS Trust): Chasty, Stewart, Chanda, Karunanithi; Royal Sussex County Hospital: Duncan, Corbett, Hill; Royal United Hospital Bath: Crowe, Wexler, Knechtli; Russells Hall Hospital: Fernandes, Neilson, Bareford, Taylor; Salford Royal Hospital: Jowitt, Houghton; Salisbury District Hospital: Cullis, Grand, Everington; Sandwell General Hospital: Hasan, Singleton Hospital: Sati, Al-Ismail, Benton; Southampton General Hospital: Richardson, Orchard, Jenner; Southern General Hospital: Morrison, Macdonald; St Bartholomew's Hospital: Oakervee; St George's Hospital: Willis, St Helier Hospital: Mercieca, Rice, Zuha, Kolade; St James's University Hospital: Bowen, Johnson, McVerry; St Richard's Hospital: Janes, Bevan; Staffordshire General Hospital: Revell; Stirling Royal Infirmary: Neilson; Stoke Mandeville Hospi- tal: Eagleton; The Alexandra Hospital: Clark; The Ayr Hospital: Eynaud; The Great Western Hospital: Gray, Blesing, Sternberg, Green; The James Cook University Hospital: Wood, Plews; The Royal Bolton Hospital: Grey; The Royal Liverpool University Hospital: Clark; The Royal Oldham Hospital: Allamedine, Elhanash, Osborne, Greenfield, Sen; The Royal Victoria Infirmary: Jackson, Jones, Lennard, O'Brien; Torbay District General Hospital: Turner, Rymes, Roberts, Smith; University College Hospital: Khwaja, Ardeshna, Yong; University Hospital Aintree: Woodcock, Sadik, Salim; University Hospital Coventry (Walsgrave): Jackson, Harrison, Bokhari, Arbuthnot, Jobanputra; University Hospital Lewisham: Mir, Yeghen; University Hospital of Wales: Poynton, Rowntree, Burnett, Kell, Knapper; Victoria Hospital: Williamson; Victoria Infirmary: Tansey, Sharp; Warwick Hospital: Borg; Western General Hospital: Roddie, Johnson; Wexham Park Hospital: Blienz, Moule, Philpott; Whiston Hospital: Nicholson, Satchi; Wishaw General Hospital: Helenglass; Worcestershire Royal Hospital: Shafeek, Pemberton; Worthing Hospital: O'Driscoll; Wycombe General Hospital: Pattinson, Kelly; York Hospital: Bond, Munro; Ysbyty Glan Clwyd: Hoyle, Heartin; Ysbyty Gwynedd District General Hospital: Seale.

\section{Funding}

Research support for the NCRI AML16 trial was provided by Cancer Research UK (grant number A6031).

\section{Data-sharing statement}

Anonymised data from the Sponsor's dataset are available to independent researchers through a standard process, which includes an internal feasibility assessment and scientific review process. Any data release is subject to participant consent and any existing contractual obligations with funders and collaborators

\section{References}

1. Burnett A, Wetzler M, Löwenberg B. Therapeutic advances in acute myeloid leukemia. J Clin Oncol. 2010;28(5):4333-4338.

2. Walter RB, Kantarjian HM, Huang X, et al. Effect of complete remission and responses less than complete remission on survival in acute myeloid leukemia: a combined Eastern Cooperative Oncology Group, Southwest Oncology Group, and M.D. Anderson Cancer Center study. J Clin Oncol. 2010;28(10):1766-1771.

3. van der Holt B, Breems DA, Berna Beverloo $\mathrm{H}$, et al. Various distinctive cytogenetic abnormalities in patients with acute myeloid leukaemia aged 60 years and older express adverse prognostic value: results from a prospective clinical trial. $\mathrm{Br} J$ Haematol. 2007;136(1):96-105.

4. Appelbaum FR, Gundacker H, Head DR, et al. Age and acute myeloid leukemia. Blood. 2006;107:3481-3485.

5. Leith CP, Chir B, Kopecky KJ, et al. Acute myeloid leukemia in the elderly: assessment of multidrug resistance (MDR1) and cytogenetics distinguishes biologic subgroups with remarkably distinct responses to standard chemotherapy. A Southwest Oncology Group study. Blood. 1997;89(9):3323-3329.

6. Burnett AK, Russell NH, Hills RK et al. Addition of gemtuzumab ozogamicin to induction chemotherapy improves survival in older patients with acute myeloid leukemia. J Clin Oncol. 2012;30(32):3924-3931.

7. Russell NH, Kjeldsen L, Craddock C, et al. A comparative assessment of the curative potential of reduced intensity allografts in acute myeloid leukaemia. Leukemia. 2015;29(7):1478-1484.

8. Cornelissen JJ, Versluis J, Passweg JR, et al. Comparative therapeutic value of post-remission approaches in patients with acute myeloid leukemia aged 40-60 years. Leukemia. 2015;29(5):1041-1050.

9. Lim Z, Brand R, Martino R, et al. Allogeneic hematopoietic stemcell transplantation for patients 50 years or older with myelodysplastic syndromes or secondary acute myeloid leukemia. J Clin Oncol. 2010;28 (3):405-411. 
10. McClune BL, Weisdorf DJ, Pedersen TL, et al. Effect of age on outcome of reduced-intensity hematopoietic cell transplantation for older patients with acute myeloid leukemia in first complete remission or with myelodysplastic syndrome. J Clin Oncol. 2010;28(11):1878-1887.

11. Sorror ML, Sandmaier BM, Storer BE, et al. Long-term outcomes among older patients following nonmyeloablative conditioning and allogeneic hematopoietic cell transplantation for advanced hematologic malignancies. JAMA. 2011;306(17):1874-1883.

12. Devine SM, Owzar K, Blum W, et al. Phase II study of allogeneic transplantation for older patients with acute myeloid leukemia in first complete remission using a reduced-intensity conditioning regimen: results from Cancer and Leukemia Group B 100103 (Alliance for Clinical Trials in Oncology)/Blood and Marrow Transplant Clinical Trial Network 0502. J Clin Oncol. 2015;33(35):4167-4175.

13 Gardin C, Pautas C, Fournier E, et al Added prognostic value of secondary $A M L$-like gene mutations in ELN intermediate-risk older AML: ALFA-1200 study results. Blood Adv. 2020;4(9):1942-1949.

14. Cheson BD, Bennett JM, Kopecky KJ, et al. Revised recommendations of the International Working Group for diagnosis, standardization of response criteria treatment outcomes, and reporting standards for therapeutic trials in acute myeloid leukemia. J Clin Oncol. 2003;21(24):4642-4649.

15. Mantel N, Byar DP. Evaluation of response-time data involving transient states: an illustration using heart-transplant data. J Am Stat Assoc. 1974;69(8):81-86.

16. Wheatley K, Brookes CL, Howman AJ, et al. Prognostic factor analysis of the survival of elderly patients with AML in the MRC AML11 and LRF AML14 trials. Br J Haematol. 2009;145(5):598-605.

17. Sorror ML, Sandmaier BM, Storer BE, et al. Comorbidity and disease status-based risk stratification of outcomes among patients with acute myeloid leukemia or myelodysplasia receiving allogeneic hematopoietic cell transplantation. J Clin Oncol. 2007;25 (27):4246-4254

18. Freeman SD, Virgo P, Couzens S, et al. Prognostic relevance of treatment response measured by flow cytometric residual disease detection in older patients with acute myeloid leukemia. J Clin Oncol. 2013;31(32):4123-4131.

19. Sorror ML, Logan BR, Zhu X, et al. Prospective validation of the predictive power of the Hematopoietic Cell Transplantation Comorbidity Index: a Center for International Blood and Marrow Transplant Research study. Biol Blood Marrow Transplant. 2015;21(8):1479-1487. 